\section{R. S. Miskovish}

\section{E. Brennen}

California Institute of Technology, Pasadena, Calif. 91125

\title{
Some Unsteady Fluid Forces on Pump Impellers
}

Spectral analyses of all the forces and moments acting on a typical centrifugal pump impeller/volute combination are presented. These exhibit shaft frequencies, blade passing frequencies, and beat frequencies associated with a whirl motion imposed on the shaft in order to measure rotordynamic forces. Among other features the unsteady thrust was found to contain a surprisingly large blade passing harmonic. While previous studies have explored the magnitudes of the steady fluid-induced radial forces and the fluid-induced rotordynamic forces for this typical centrifugal pump impeller/volute combination, this paper presents information on the steady bending moments and rotordynamic moments due to the fluid flow. These imply certain axial locations for the lines of action of the radial and rotordynamic forces. Data on the lines of action are presented and allow inferences on the sources of the forces.

\section{Introduction}

In previous publications (Chamieh et al., 1985, Jery et al., 1985, Adkins and Brennen 1986, Franz et al., 1990) considerable information has been presented on the flow induced radial and rotordynamic forces in centrifugal and axial (Arndt and Franz, 1986) flow pumps. The effect of cavitation on these forces has also been explored (Franz, 1989). The forces in question can be visualized by reference to Fig. 1, which is in a plane perpendicular to the axis of rotation. Steady radial forces which result from asymmetries in the volute, the discharge flow or the inflow are denoted by $F_{o x}^{*}$ and $F_{o y}^{*}$. If, in addition, the axis of rotation undergoes a small displacement given by $x(t), y(t)$ from some mean position then the instantaneous fluid forces on the impeller, $F_{x}^{*}(t)$ and $F_{y}^{*}(t)$, can be expressed by

$$
\left\{\begin{array}{l}
F_{x}^{*}(t) \\
F_{y}^{*}(t)
\end{array}\right\}=\left\{\begin{array}{l}
F_{0 x}^{*} \\
F_{0 y}^{*}
\end{array}\right\}+\left[A^{*}\right]\left\{\begin{array}{l}
x(t) \\
y(t)
\end{array}\right\}
$$

where $\left[A^{*}\right]$ is the rotordynamic matrix. In addition to the radial forces, the corresponding bending moments should be divided into steady radial moments and a rotordynamic moment matrix as follows:

$$
\left\{\begin{array}{l}
M_{x}^{*}(t) \\
M_{y}^{*}(t)
\end{array}\right\}=\left\{\begin{array}{l}
M_{o x}^{*} \\
M_{o y}^{*}
\end{array}\right\}+\left[B^{*}\right]\left\{\begin{array}{l}
x(t) \\
y(t)
\end{array}\right\}
$$

The forces and moments are presented in dimensionless form denoted by the same symbols without the asterisk. The steady radial forces and moments are nondimensionalized by $\rho \pi \Omega^{2} r_{2}^{3} b_{2}$ and $\rho \pi \Omega^{2} r_{2}^{4} b_{2}$ respectively, the matrices $\left[A^{*}\right]$ and $\left[B^{*}\right]$ by $\rho \pi \Omega^{2} r_{2}^{2} b_{2}$ and $\rho \pi \Omega^{2} r_{2}^{3} b_{2}$ and the displacements are non-dimensionalized by $r_{2}$. If for simplicity we focus on a circular whirl orbit (see Fig. 1) of eccentricity $\epsilon$ and frequency $\omega$, then $x=\epsilon \cos \omega t$ and $y=\epsilon \sin \omega t$, and

Contributed by the Fluids Engineering Division for publication in the JoURNAL of FluIDs ENGINEERING. Manuscript received by the Fluids Engineering Division April 16, 1991. Associate Technical Editor: U. S. Rohatgi.

$$
\begin{aligned}
\left\{\begin{array}{l}
F_{x}(t) \\
F_{y}(t)
\end{array}\right\} & =\left\{\begin{array}{l}
F_{o x} \\
F_{o y}
\end{array}\right\}+\epsilon[A]\left\{\begin{array}{l}
\cos \omega t \\
\sin \omega t
\end{array}\right\} \\
\left\{\begin{array}{l}
M_{x}(t) \\
M_{y}(t)
\end{array}\right\} & =\left\{\begin{array}{l}
M_{o x} \\
M_{o y}
\end{array}\right\}+\epsilon[B]\left\{\begin{array}{l}
\cos \omega t \\
\sin \omega t
\end{array}\right\}
\end{aligned}
$$

Virtually all of the previous experimental data confirms the reasonable supposition that the matrices $[A]$ and $[B]$ should be independent of the particular choice of axes, $x$ and $y$. Such rotational invariance requires that

$$
\begin{aligned}
& A_{z z}=A_{y y}=F_{n} ; A_{y x}=-A_{x y}=F_{t} \\
& B_{x x}=B_{y y}=M_{n} ; B_{y x}=-B_{x y}=M_{t}
\end{aligned}
$$

where $F_{n}, F_{t}, M_{n}, M_{t}$ are the rotordynamic forces and moments normal and tangential to the whirl orbit non-dimensionalized by $\rho \pi \Omega^{2} r_{2}^{2} b_{2} \epsilon$ in the case of the forces and by $\rho \pi \Omega^{2} r_{2}^{3} b_{2} \epsilon$ in the

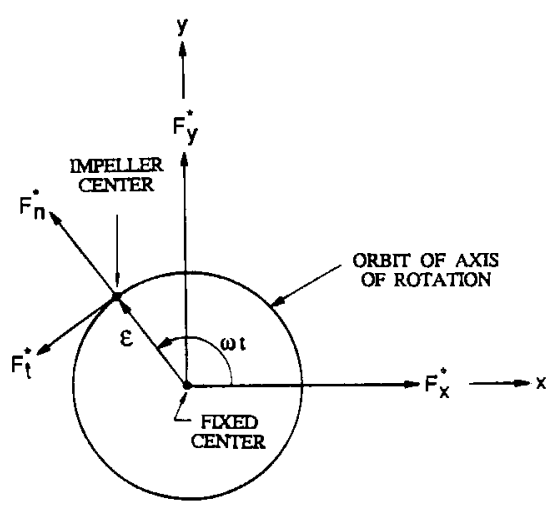

Fig. 1 Schematic defining the radial forces $F_{x}^{*}$ and $F_{y}^{*}$ and the rotordynamic forces $F_{n}^{*}$ and $F_{f}^{*}$ in a plane perpendicular to the axis of rotation of the impeller. The instantaneous position of the impeller center and its locus on a circular whirl orbit are shown. 
case of the moments. Like $[A]$ and $[B]$, the rotordynamic forces and moments will be functions of the whirl frequency and mean operating condition but independent of $\epsilon$ if the displacement is within a linear regime. Furthermore, presentation of $F_{n}, F_{r}, M_{n}, M_{f}$ specifies the entire matrices and has the advantage of ease of physical interpretation. For example, if $F_{t}$ is in the same direction as the whirl motion, then it represents a fluid-induced destabilizing force from a rotordynamic perspective.

\section{Spectra of Forces on a Centrifugal Impeller}

During the present investigation a comprehensive examination was undertaken of all the unsteady fluid-induced forces and moments acting upon a typical centrifugal pump impeller. The specific impeller and volute was that combination designated Impeller X/Volute A (see Jery et al., 1985). Impeller X is a five-bladed centrifugal pump with a discharge radius of $8.1 \mathrm{~cm}$. and a design specific speed of 0.57 made by Byron Jackson Pump Company. Volute $A$ is a vaneless, spiral volute made to match Impeller $X$ at a flow coefficient $\phi=0.092$.

A sketch of the volute and of the impeller mounting is included in Fig. 2. Note that since the impeller moves on an eccentric orbit the clearances between the impeller and the volute vary with time.

The forces described in the preceding section were evaluated from measurements of the radial forces, $F_{1}$ and $F_{2}$, and moments, $M_{1}$ and $M_{2}$, in the coordinate frame of the shaft-mounted rotating balance to which the impellers were directly mounted. This balance measured the unsteady components of all six forces and moments, including the torque $M_{3}$, and the axial thrust, $F_{3}$.

As part of the present investigation, the dynamic signals associated with all six forces and moments during operation of the pump at a particular flow coefficient, $\phi$, shaft frequency, $\Omega$, and whirl frequency, $\omega$, were closely examined and revealed at least one surprise which we shall come to shortly. To discuss the results (of which Fig. 3 represents a typical example), it is necessary to describe briefly how the motions are controlled in the experiment. A generator produces a low frequency signal denoted by $\Omega / J$ where $J$ is an integer typically 10 or 20 . This is then multiplied by $J$ to produce the signal which drives the main shaft motor. The basic signal is also multiplied by a second integer, $I$, and this is used to drive the whirl motion so that $\omega=I \Omega / J$. Both motions are closely controlled and monitored as part of the data acquisition system. It follows that in the frame of reference of the rotating balance the radial
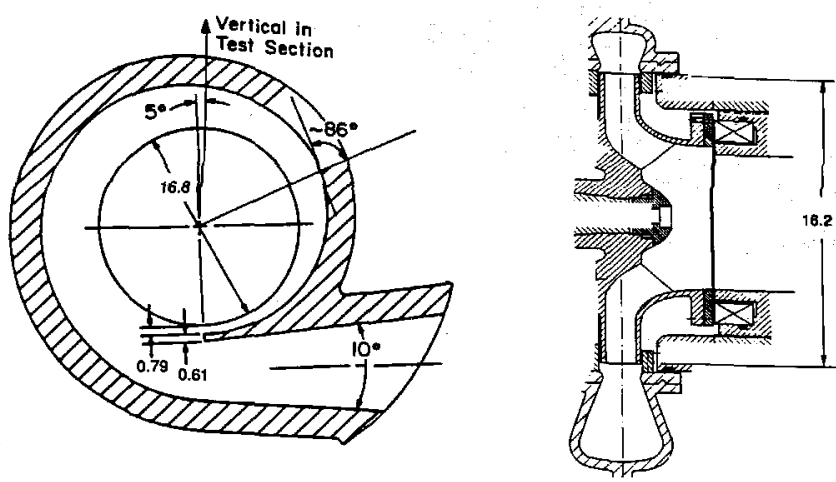

Fig. 2 Sketches of Volute $A$ (left) and Impeller $X$ mounted in the facility (right). Note sketches are two different scales (dimensions in $\mathrm{cm}$.).

forces and moments which are steady in the laboratory frame will show up at the frequency, $\Omega$. The rotordynamic forces and moments will be represented by signals at frequencies of $(\Omega \pm$ $\omega)$ or $\Omega(J \pm I) / J$. Blade passage frequencies will also be manifest; in the case studied here the Impeller $X$ had five blades and, in combination with the vaneless volute, would generate a blade passage signal at $5 \Omega$. Fourier components were obtained by cross-correlation for all integer multiples of $\Omega / J$ up to about $6 \Omega$; tests revealed no significant amplitudes above this frequency.

Spectra like this were obtained at a number of shaft speeds, whirl ratios, $I / J$, and operating conditions represented by the flow coefficient, $\phi$. They all had similar characteristics exemplified by Fig. 3, in which the results for each of the six forces and moments are normalized with respect to the maximum component occurring in that particular spectrum. Those normalizing values are attached to each spectrum and should be compared with the later data of Figs. 6, 7, and 8 in order to assess whether the amplitudes are significant or not. The following conclusions can be drawn from Fig. 3 and other similar spectra:

(a) The strong peak at $\Omega$ in all of the radial forces and bending moments $\left(F_{1}, F_{2}, M_{1}\right.$, and $\left.M_{2}\right)$ is generated by steady radial forces and bending moments due to the asymmetry of the volute.

(b) The peaks at $(J \pm I) \Omega / J$ in $F_{1}, F_{2}, M_{1}$, and $M_{2}$ are generated by nonzero $F_{n}, F_{t}, M_{n}$, and $M_{t}$. It is particularly noticeable that the $(J-I) \Omega / J$ component is usually much larger than the $(J+I) \Omega / J$ component and hence

\section{Nomenclature}

\footnotetext{
$A_{2}=$ impeller discharge area $=2 \pi r_{2} b_{2}$

$b_{2}=$ impeller discharge width

$F_{1}, F_{2}=$ radial forces on the impeller observed in the rotating frame of the balance

$F_{3}=$ thrust on the impeller

$F_{o x}, F_{o y}=$ normalized mean radial forces acting on the impeller where $x$ is the direction of the volute cutwater and $y$ is a perpendicular direction rotated in the direction of shaft rotation. Unless otherwise stated, all forces are normalized
}

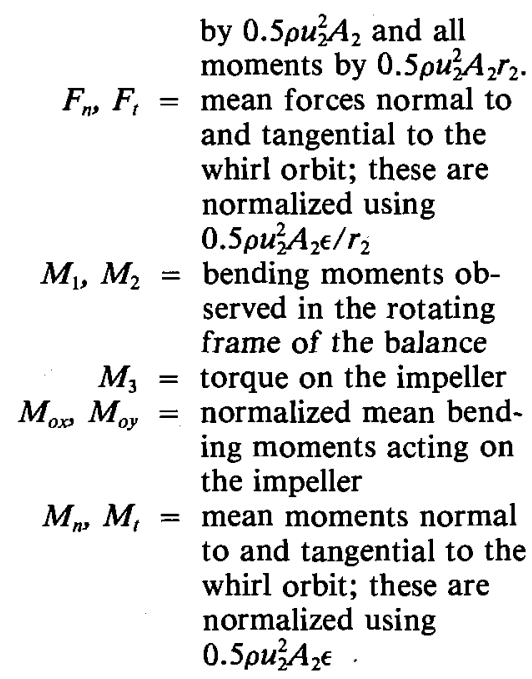

$$
\begin{aligned}
& r_{2}=\text { impeller discharge ra- } \\
& \text { dius } \\
& u_{2}=\text { impeller tip speed at } \\
& \text { discharge }=\mathbf{\Omega} r_{2} \\
& \epsilon=\text { radius of the eccentric } \\
& \text { whirl orbit } \\
& \phi=\text { flow coefficient }=Q^{\prime} \\
& u_{2} A_{2} \text {, where } Q \text { is the } \\
& \text { volume flow rate } \\
& \text { through the pump } \\
& \psi=\text { head coefficient }= \\
& g \Delta h / u_{2}^{2} \text {, where } \Delta h \text { is the } \\
& \text { total head rise across } \\
& \text { the pump } \\
& \Omega=\text { pump rotational speed } \\
& (\mathrm{rad} / \mathrm{s}) \\
& \omega=I \Omega / J=\text { whirl rotational speed } \\
& (\mathrm{rad} / \mathrm{s}) \text { where } I \text { and } J \\
& \text { are integers } \\
& \omega / \Omega=I / J=\text { whirl ratio }
\end{aligned}
$$



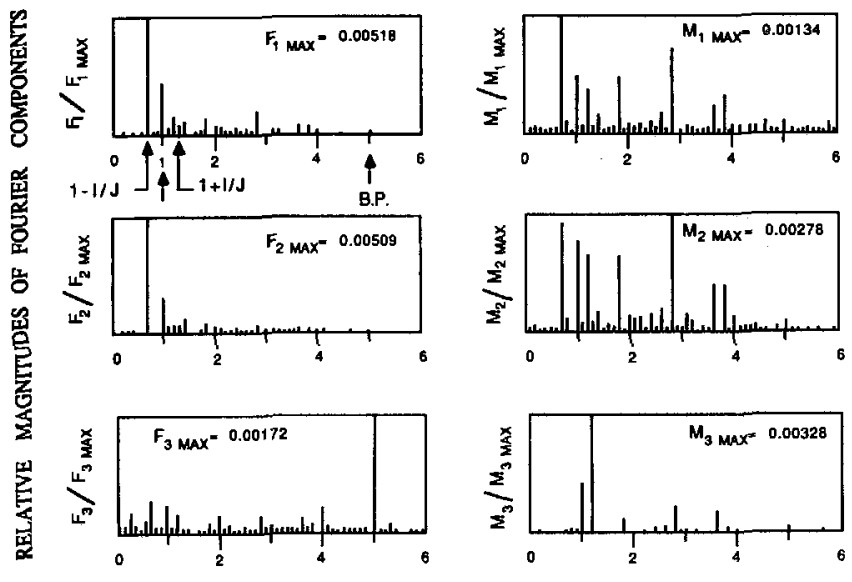

FREQUENCY / SHAFT FREQUENCY

Fig. 3 Typical frequency content of $F_{1}, F_{2}, F_{3}, M_{1}, M_{2}, M_{3}$ for Impeller $X /$ Nolute $A$ for tests at $3000 \mathrm{rpm}, \varphi=0.092$, and $I J=3 / 10$. Note the harmonics $\Omega,\left(J_{ \pm}\right) \Omega / J$ and the blade passing frequency, $5 \Omega$. Uncertainty in force components is $\pm \mathbf{0 . 0 0 0 5}$, in moment components is $\pm \mathbf{0 . 0 0 0 3}$.

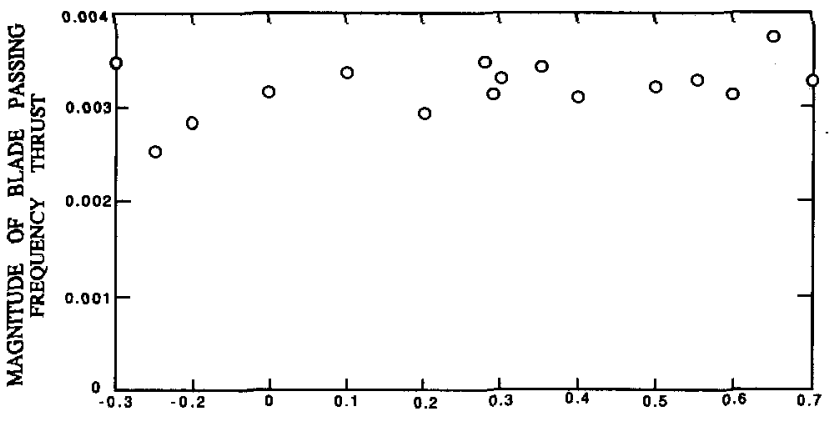

WHIRL FREQUENCY RATIO, $\omega / \Omega$

Fig. 4 The blade passing frequency component of thrust oscillation as a function of whirl frequency ratio, $\omega / \Omega$, for Impeller $X /$ olute $A$ op. erating at $3000 \mathrm{rpm}$ and $\varphi=0.092$. Uncertainty in ordinate is $\pm \mathbf{0 . 0 0 0 5}$.

the former frequency provides the major contribution to $F_{n}, F_{t}, M_{n}, M_{t}$, and the rotordynamic matrices.

(c) The moments $M_{1}$ and $M_{2}$ seem substantially noisier than the forces $F_{1}$ and $F_{2}$. This is rather misleading; it is due to the fact that the moments are small since the origin of the force/moment coordinate system is in the center of the discharge of the impeller and the line of action of the radial forces is close to this axial location.

(d) The unsteady axial thrust, $F_{3}$ contains a surprisingly large component at the blade passing frequency, $5 \Omega$, though other multiples of the impeller rotation frequency are also present. Note that since the balance only records unsteady forces, neither the steady thrust or torque can be measured by this device.

(e) The unsteady torque, $M_{3}$, was usually quite small compared with the steady torque which was estimated using separate strain gauges on the drive shaft. As demonstrated by Fig. 3, no consistent pattern was observed in the frequency content of this unsteady torque. In all cases, the largest harmonic is $60 \mathrm{~Hz}$, which corresponds to frequency of the electrical input to the balance. In light of the blade passing frequency content in the thrust, one might have expected a similar contribution to the torque. However this was not observed.

\section{Unsteady Thrust at Blade Passage Frequency}

In the previous section we have noted the significant component in the unsteady thrust at the blade passing frequency.
In this section we shall examine the relative magnitude of this unsteady force. Since the balance does not record the steady thrust and since we do not have independent means of measuring the thrust, we shall compare the magnitude of the measured unsteady thrust with an estimate of the steady thrust based on measurement of the pressure rise across the pump and of the pressure distribution around the impeller.

From the geometry of the five-bladed centrifugal pump impeller, Impeller X, and assuming (i) that the discharge pressure acts on the back-face of the impeller and (ii) that the pressure on the exterior of the shroud varies linearly with radial position between the inlet tip and the discharge tip, we find by integration of the pressure distribution on the impeller that

$$
\frac{\text { Mean Thrust }}{0.5 \rho u_{2}^{2} A_{2}}=F_{03}=3.09 \phi^{2}-a \psi
$$

in which $a=3.0$. This provides values which are acceptably close to those of Beitz and Küttner (1981) who recommend a value of $a$ in the range 3.6-4.6. With this estimate of steady thrust, ratios of the magnitude of the unsteady thrust at the blade passing frequency to the steady thrust were evaluated for a range of flow coefficients, $\phi$, and whirl ratios. With respect to the latter, Fig. 4 demonstrates that, as expected, the ratio is independent of the whirl ratio since the interaction mechanism occurs irrespective of whirl. Indeed Fig. 4 merely indicates the typical scatter in the magnitude of the unsteady thrust.

The variation with operating point is exemplified by the values in the following table:

Table 1 Steady and unsteady thrust

\begin{tabular}{ccccc}
\hline \multicolumn{2}{l}{ Operating point } & Steady thrust & $\begin{array}{c}\text { Unsteady } \\
\text { thrust magnitude/ } \\
\text { steady thrust }\end{array}$ & \multicolumn{1}{c}{$\theta_{m}$} \\
\hline$\phi$ & $\psi$ & $F_{03}$ & 0.0069 & $-17^{\circ}$ \\
\hline 0.060 & 0.510 & -1.52 & 0.525 & $17^{\circ}$ \\
0.092 & 0.425 & -1.25 & 0.0028 & $23^{\circ}$ \\
0.104 & 0.375 & -1.09 & 0.0065 &
\end{tabular}

The nominal design flow coefficient of this impeller/volute combination is 0.092 . Note that, as might be expected, the unsteady thrust at the blade passing frequency is smallest at the design flow coefficient. Though the magnitude of unsteady thrust is less than one percent of the steady thrust, it is possible to envisage circumstances in which it could cause serious axial resonance problems.

Finally, the relative location of the impeller blades to the volute cutwater at the time of the peak thrust is of interest in attempting to understand the origins of this unsteady thrust. Since Impeller $X$ is five-bladed the angle between the tips of the five blades is $72 \mathrm{deg}$. If we define an angle between the line from the impeller center to the volute cutwater and the line from the impeller center to the discharge blade tip at the time when the instantaneous thrust is a maximum then this angle would take values of $\theta_{m}^{*}=\theta_{m} \pm 72 n \mathrm{deg}(n=0,1,2$, etc.) where the observed values of $\theta_{m}$ are as listed in Table 1 . The positive direction of $\theta_{m}$ is defined as being in the direction of rotation of the impeller. Note that in all three cases listed in Table 1 the maximum instantaneous thrust occurs when the discharge tip of a blade is close to the volute cutwater.

\section{Origins of the Radial and Rotordynamic Forces}

Previous publications, (Chamieh et al., 1985; Jery et al., 1985; Adkins and Brennen, 1988; Franz et al., 1990) have presented data on the steady radial forces and on the rotordynamic forces for a number of impellers, diffusers and volutes. Visualizing the centrifugal pump impeller as a control volume, one can recognize that there exists the possibility of contributions to the radial force from three different sources. First, circumferential variation in the impeller discharge pres- 

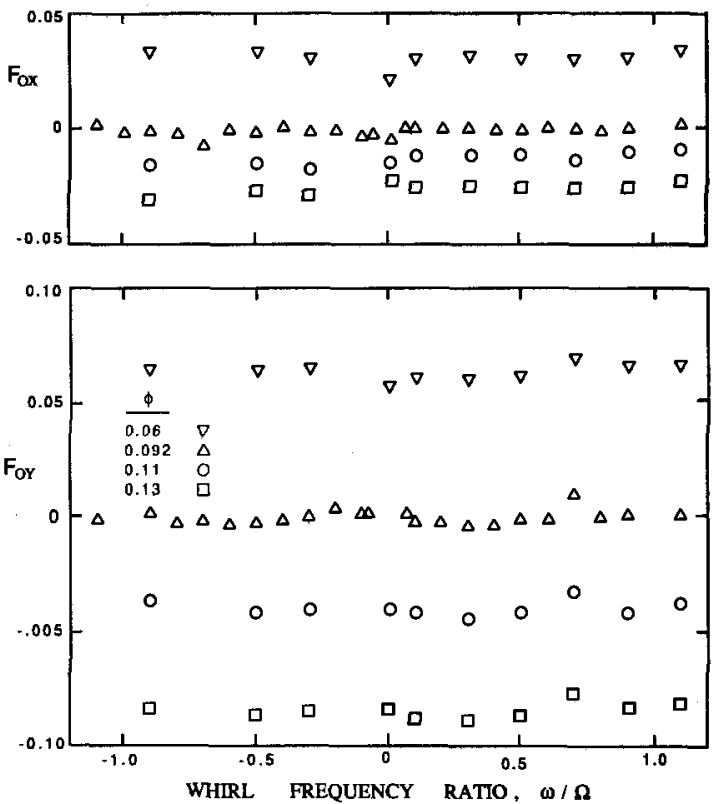

Fig. 5 Steady radial forces, $F_{o x}$ and $F_{o y}$ for Impeller X/Volute $A$ at a speed of $1000 \mathrm{rpm}$ and various flow coefficients. Uncertainty expressed as a standard deviation: $F_{\text {ox }} F_{\text {ay }} \pm 0.0008$.
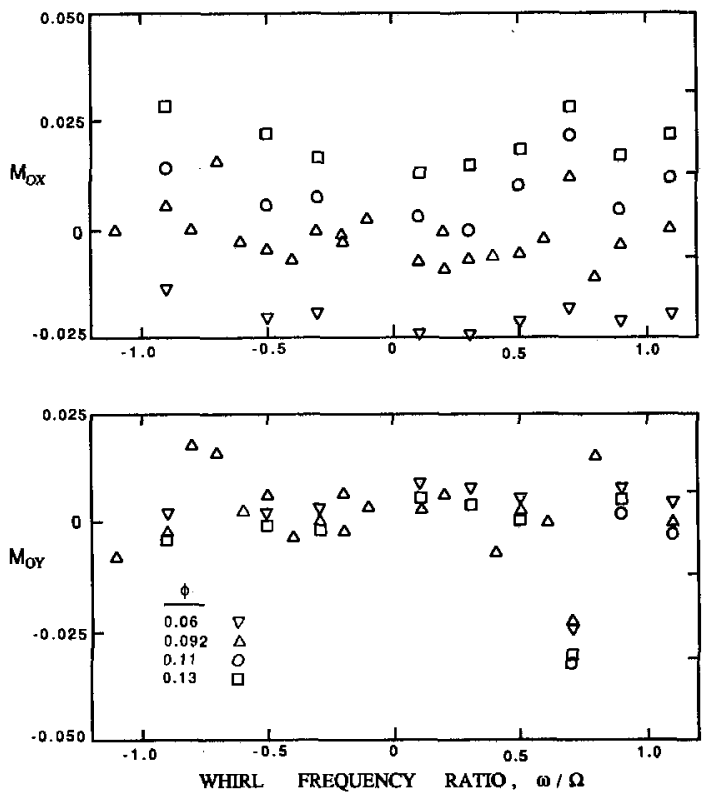

Fig. 6 Steady radial moments, $\boldsymbol{M}_{o x}$ and $\boldsymbol{M}_{\text {oy, }}$ for Impeller X/Volute A at a speed of $1000 \mathrm{rpm}$ and various flow coefficients. Uncertainty expressed as a standard deviation: $M_{o x}, M_{o y} \pm 0.0005$.

sure (or volute pressure) will clearly result in a radial force acting on the impeller discharge area. A second contribution could be caused by the leakage flow from the impeller discharge to the inlet between the impeller shroud and the pump casing. Circumferential nonuniformity in the discharge pressure could cause circumferential nonuniformity in the pressure within this shroud/casing gap and therefore a radial force acting on the exterior of the pump shroud. For convenience we shall term this second contribution the leakage flow contribution. Thirdly, a circumferential nonuniformity in the flow rate out of the impeller would imply a force due to the nonuniformity in the momentum flux out of the impeller. Adkins and Brennen (1988) used an impeller flow model in conjunction with a one-dimensional volute flow model to predict the circumferential non-uniformity in the pressure acting on the impeller discharge and found that these pressure distributions were in accord with experimental measurements. Also, integration of the experimental pressure distributions yielded radial forces in good agreement with both the overall radial forces measured using the force balance and the theoretical predictions of the theory. These results demonstrate that it is primarily the circumferential non-uniformity in the pressure at the impeller discharge which generates the radial forces, but that the non-uniformity in the pressures acting on the exterior of the shroud may also contribute.

The origins of the rotordynamic forces were also explored by Adkins and Brennen (1988) who used the same model to evaluate the rotordynamic forces acting on the impeller discharge. It was found that the theoretical values for the impeller discharge contributions were significantly smaller than the total measured forces. Thus it was concluded that the leakage flow around the shroud exterior can be an important contributor to the rotordynamic forces. To confirm this, Adkins and Brennen (1988) made experimental measurements of the pressure distributions in both the impeller discharge flow and in the leakage flow.

None of these previous experimental or theoretical studies addressed the issue of the location of the lines of action of either the radial or the rotordynamic forces. Clearly, a discussion of the lines of action or of the moments requires a definition of the axial location of the origin of the reference frame of the forces and moment. In this paper we arbitrarily choose the origin to be in the center of the impeller discharge. If the radial forces $F_{o x}, F_{o y}$ acted at this location, then the moments $M_{o x}$ and $M_{o y}$ would be zero. It is one of the purposes of this paper to present data on both the steady radial moments and the rotordynamic moments and in doing so to provide information of the location of the lines of action of the radial and rotordynamic forces.

\section{Forces and Moments}

Typical data on the radial forces and moments are presented in Figs. 5 and 6, respectively, for the Impeller X/Volute A combination at a shaft speed of $1000 \mathrm{rpm}$ and four different flow coefficients. Note that the forces are independent of the whirl ratio as they should be. The data of Fig. 5 correspond to data presented previously by Jery (1987). The moments in Fig. 6 are new. It should be noted that they follow a righthand rule in which the $z$-axis points toward the inlet. Thus a radial force in the $x$ or $y$ direction whose line of action is closer to the inlet than the origin in the center of the impeller discharge, leads to a positive $M_{o y}$ or a negative $M_{o x}$. Though the data in Fig. 6 contains a few anomalous points, it does suggest that there exists a steady moment, primarily in the $x$-direction and that this changes with the flow coefficient. The following example illustrates the typical magnitude of these steady moments. At a flow coefficient of $\phi=0.06$, the steady force vector $\mathbf{F}_{o}=F_{o x} \mathbf{x}+F_{o y} \mathbf{y}$ has components $F_{o x} \approx 0.03$ and $F_{o y}$ $\approx 0.06$ (see Fig, 5); it has a magnitude of about 0.067 and an angle from the $x$-axis, $\theta_{f}$, of $63 \mathrm{deg}$. The steady moment vector $\mathbf{M}_{o}=M_{o x} \mathbf{x}+M_{o y} \mathbf{y}$, where $M_{o x} \approx-0.02$ and $M_{o y} \approx 0$, has a magnitude of 0.02 and an angle from the $x$-axis, $\theta_{m}$, of 180 deg. The position of the line of action on the $z$-axis can be computed from $\mathbf{r} * \mathbf{F}=\mathbf{M}$, (or, in scalar terms $F * r=M \perp$, where $M \perp=M * \sin \left(\theta_{m}-\theta_{f}\right)$ ), and the resulting value of $r$ when $\phi=0.06$ is 0.27 or $2.2 \mathrm{~cm}$ (compared with an impeller discharge radius of $8.1 \mathrm{~cm}$ ). Thus the line of action in this instance is $2.2 \mathrm{~cm}$ forward from the center of the discharge. For $\phi=0.13$, a similar estimate gives a location $1.3 \mathrm{~cm}$ forward of the center of the discharge.

In conclusion, the steady moments indicate that the line of action of the steady radial force is some distance ahead of the center of the discharge, though it moves backward with in- 

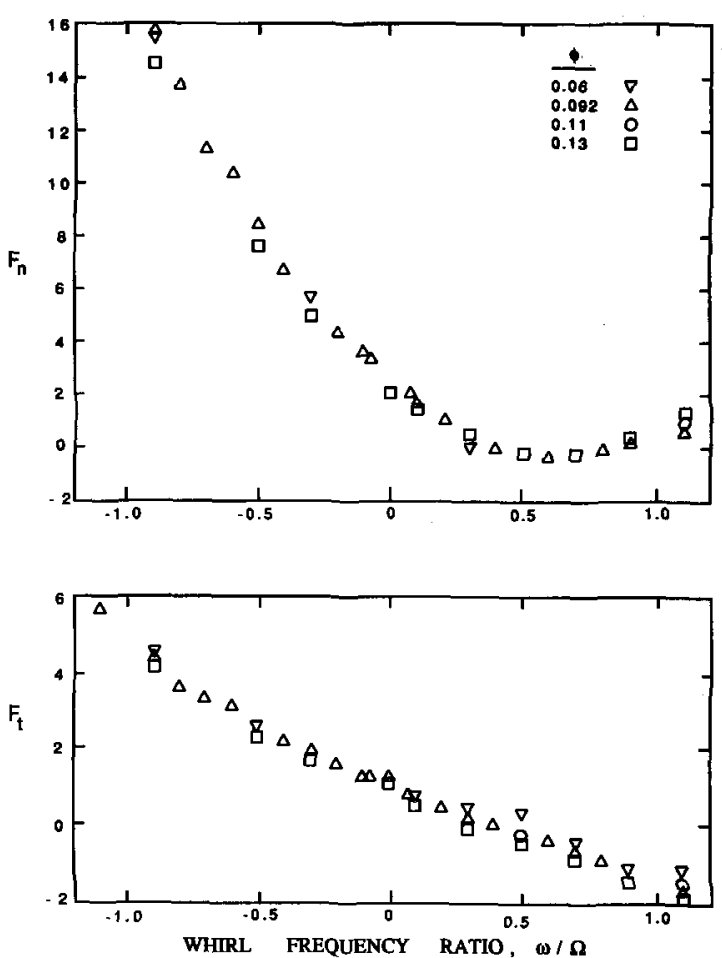

Fig. 7 Normal and tangential rotordynamic forces, $\boldsymbol{F}_{n}$ and $\boldsymbol{F}_{\mathrm{b}}$ for $1 \mathrm{~m}$ peller X/Volute $A$ at $1000 \mathrm{rpm}$ and various flow coefficients as in Figs. 5 and 6. Uncertainty expressed as a standard deviation: $F_{m} F_{1} \pm 0.05$.
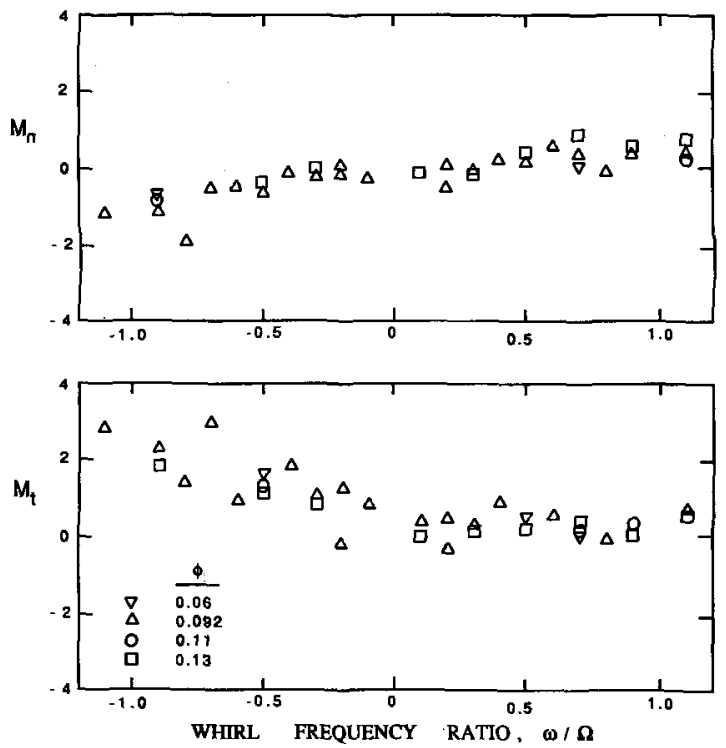

Fig. 8 Normal and langential rotordynamic moments, $M_{n}$ and $M_{t}$, for Impeller X/Volute $A$ at $1000 \mathrm{rpm}$ and various flow coefficients as in Figs. 5 and 6. Uncertainty expressed as a standard deviation: $M_{m} M_{t} \pm \mathbf{0 . 1}$.

creasing flow coefficient. The reason the lines of action are forward of the impeller discharge is the contribution to the steady radial force from the pressure distribution on the exterior of the shroud which combines with the force on the discharge to yield the total steady radial force. Since the force on the exterior of the shroud is comparable with that on the discharge, it follows that the line of action of the combination will be forward of the center of the discharge. The present observations are consistent with that previous analysis.

Now we examine the rotordynamic moment matrices. Figure 8 indicates that the values of $M_{n}$ and $M_{t}$ are very small and this accounts for the larger scatter in this data. The location of the lines of action of $F_{n}$ and $F_{t}$ relative to the center of the discharge can be computed in a manner similar to that used for computation of the lines of action of the steady forces. Typical values from Figs. 7 and 8 indicate that these displacements are much smaller than for $F_{o x}$ and $F_{o y}$ and are less than $1 \mathrm{~cm}$. Thus we conclude that the line of action of the rotordynamic force matrix is close to the center of the discharge. This, too, is consistent with previous analysis which suggest that for the impeller/volute combination used in these experiments the shroud force contributions to the rotordynamic matrices are quite small.

\section{Conclusions}

The conclusions drawn from this study of the fluid forces on a typical centrifugal impeller are:

(i) The spectral analysis of the forces shows the largest peaks at the frequencies $\Omega$ and $\Omega \pm \omega$ for $F_{1}, F_{2}, M_{1}$, and $M_{2}$. These frequencies correspond to the steady forces and moments, and the rotordynamic force and moments respectively. In addition, the spectra of the unsteady thrust was found to contain a strong harmonic at the blade passing frequency.

(ii) On closer examination, the magnitude of the unsteady thrust at blade passing frequency is shown to be less than one percent. Although small, this component of the unsteady thrust could lead to axial resonance problems. It has also been shown that the maximum instantaneous thrust occurs when the discharge tip of a blade is near the volute cutwater.

(iii) The steady radial moments are equivalent to a line of action for the steady radial forces which is as much as 0.25 radii from the center of the impeller discharge (radius $8.1 \mathrm{~cm}$ ) in the direction of the inlet. This is consistent with the conclusion of Adkins and Brennen (1988) that the forces on the shroud provide important contributions to the radial forces. The line of action moves closer to the center of impeller discharge as the flow coefficient increases.

(iv) The rotordynamic moment matrices are close to the rotationally invariant form that has been used to describe the rotordynamic force matrix. Thus, it suffices to present the moment matrices in terms of $M_{n}$ and $M_{t}$ without loss of information. In comparing the steady and unsteady moments with the steady and unsteady forces, the line of action in the $z$-direction is shown to be close to the center of the impeller discharge (within about 0.1 radii).

\section{Acknowledgments}

This work was performed under Contract NAS 8-33108 from NASA George Marshall Space Flight Center, Huntsville, Alabama; the authors are very grateful for their support. We should also like to express our thanks to R. Franz, N. Arndt, A. J. Acosta, and T. K. Caughey for their help during the conduct of this research program.

\section{References}

Adkins, D. R., 1986, "Analysis of Hydrodynamic Forces on Centrifugal Pump Impellers," Ph.D. Thesis, Division of Engineering and Applied Science, California Institute of Technology.

Adkins, D. R., and Brennen, C. E., 1988, “Analysis of Hydrodynamic Radial Forces on Centrifugal Pump Impellers," ASME Journal of Fuutos EngineERING, Vol. 110, pp. 20-28.

Arndt, N., and Franz, R., 1986, "Observations of Hydrodynamic Forces on Several Inducers Including the SSME-LPOTP," California Institute of Technology, Division of Engineering and Applied Science, Rep. No. E249.3.

Beitz, W., and Küttner, K.-H. (eds.), I981, Dubbel: Tachenbuch für den Maschinenbau Springer-Verlag, Heidelberg, pp. 897-899.

Brennen, C. E., Acosta, A. J., and Caughey, T. K., 1986, "Impeller Fluid Forces," Proceedings of Advanced Earth-to-Orbit Propulsion Technology Congress, Huntsville, AL, May, NASA Conf. Publ. 2436, pp. 270-295. 
Chamieh, D. S., 1983, "Forces on a Whirling Centrifugal Pump-Impeller," Ph.D. thesis, Division of Engineering and Applied Science, California Institute of Technology.

Chamieh, D., Acosta, A. J., Brennen, C. E., and Caughey, T. K., 1985, "Experimental Measurements of Hydrodynamic Radial Forces and Stiffness Matrices for a Centrifugal Pump-Impeller," ASME JourNal of Fluids ENGINEERING, Vol. 107, No. 3, pp. 307-315.

Franz, R., Arndt, N., Caughey, T. K., Brennen, C. E., and Acosta, A. J., 1987, "Rotordynamic Forces on Centrifugal Pump Impellers," Proceedings of the Eighth Conference on Fluid Machinery, Akademiai Kiado, Budapest, Vol. 1, pp. 252-258.

Franz, R, 1989, "Experimental Investigation of the Effect of Cavitation on the Rotordynamic Forces on a Whirling Centrifugal Pump Impeller,' Ph.D. thesis, Division of Engineering and Applied Science, California Institute of Technology.

Franz, R., Acosta, A. J., Brennen, C. E., and Caughey, T. K., 1990, "The
Rotordynamic Forces on a Centrifugal Pump Impeller in the Presence of Cavitation," ASME Journal of FluIDs ENGINEERING, Vol. 112, No. 3, pp. 264271

Jery, B., and Franz, R., 1982, "Stiffness Matrices for the Rocketdyne Diffuser Volute," Report No. E249.1, California Institute of Technology.

Jery, B., Acosta, A. J., Brennen, C. E., and Caughey, T. K., 1985, "Forces on Centrifugal Pump Impellers," Second International Pump Symposium, Houston, TX, April 29-May 2.

Jery, B., Acosta, A. J., Brennen, C. E., and Caughey, T. K., 1986, "Hydrodynamic Impeller Stiffness, Damping and Inertia in the Rotordyammics of Centrifugal Punıs," Third Workshop on Rotordynamics Instability Problems in High Performance Turbomachinery, Texas A\&M University, NASA Conference Publication 2338, pp. 137-160.

Jery, B., 1987, "Experimental Study of Unsteady Hydrodynamic Force Matrices on Whirling Centrifugal Pump Impellers," Ph.D. thesis and Report No. 200.22, California Institute of Technology.

\section{For Your ASME Bookshelf}

\section{Measurement and Modeling of Environmental Flows 1992}

Editors: S.A. Sherif, D.E. Stock, E.E. Michaelides, L.R. Davis, I. Celik, B. Khalighi, R. Kumar

Topics include: mass flow measurement of gaseous breathing air through the liquid air pack respirator, quantitative flow measurement and numerical simulation of lid-driven rotating cavity flow, interfacial transport in river-reservoir systems; numerical prediction of a turbulent plume in a stably stratified environment; effects of coastal topography on lake flows; effects of viscosity on gravity currents in the inertial regime; lee waves, benign and malignant; more.

\section{ISBN 0-7918-1128-X FED-Vol. 143/HTD-Vol. $232 \quad 252$ pp.}

\section{Order No. G00772 \$62.50 List/\$50 ASME Members}

To order write ASME Information Central, 22 Law Drive, Box 2300, Fairfield, NJ 07007-2300 or call 800-THE-ASME (843-2763) or tax 201-882-1717. 